\title{
Infrared thermographic nondestructive testing of defects between aluminum panel and foam core
}

\author{
Liu Yingtao, Guo Guangping, Yang Danggang, Tang Jia and Li Shuoning \\ Beijing Institute of Aeronautical Materials, Beijing, China, yingt.liu@163.com
}

\begin{abstract}
To find debonding defects between aluminum panel and foam core in components, a sample was designed and manufactured, in which there were a few debonding defects designed. Signals of debonding defects in the sample was analyzed and used to be compared with those of suspicious areas in the component. Some areas in the component were dissected to prove results. Results of infrared thermographic non-destructive testing of the component before and after load test were compared. Influence factors of defect identification were analyzed, such as defect size, defect thickness, defect depth, curve surface of component, black paint thickness and uniformity. A conclusion was made that infrared thermographic non-destructive testing was suited to test debonding defects in that kind of structure.
\end{abstract}

Keywords: composite materials, infrared thermographic nondestructive testing, debonding defects, pulsed thermography, foam core

\section{Introduction}

Composite materials have been used more and more in aeronautics and astronautics with improvement of design level and processing technique of composite materials. Foam core sandwich structure is one kind of important composite materials due to its light weight, low thermal conductivity, and easy for large parts manufacture. Debonding defects between panel and foam core are one sort of common defects in this kind of components. It is important to study on nondestructive testing of those defects because it's difficult to find one effective NDT method in some situation due to complex structure of panel, large thickness of foam core or special shape of component.

Infrared thermographic nondestructive testing (IT NDT) is increasingly used in the inspection of materials and structures due to its full field, explicit result, rapid and noncontact testing capabilities. In this paper, to find debonding defects between aluminum panel and foam core in a sort of component, a lot of study on IT NDT were carried out, including defect identity, dissection and verification, comparison with ultrasonic testing results, comparison with results after load test. Influence factors of defect identification were analyzed such as defect size, defect thickness, defect depth, curve surface of component, black paint thickness and uniformity.

\section{Principle}

Flash thermography was used as one method of IT NDT in this paper. In FT, a brief pulse of light energy from a flash lamp array heats the inspection surface of a specimen, and an IR camera monitors the surface temperature (or radiance) as the sample cools. Internal thermal discontinuities modify the local cooling of the surface, and the corresponding radiation flux from the surface that is detected by the IR camera [1]. The IR images were displayed and manipulated using a computer. The internal thermal discontinuities, such as voids and delaminations, were identified. 


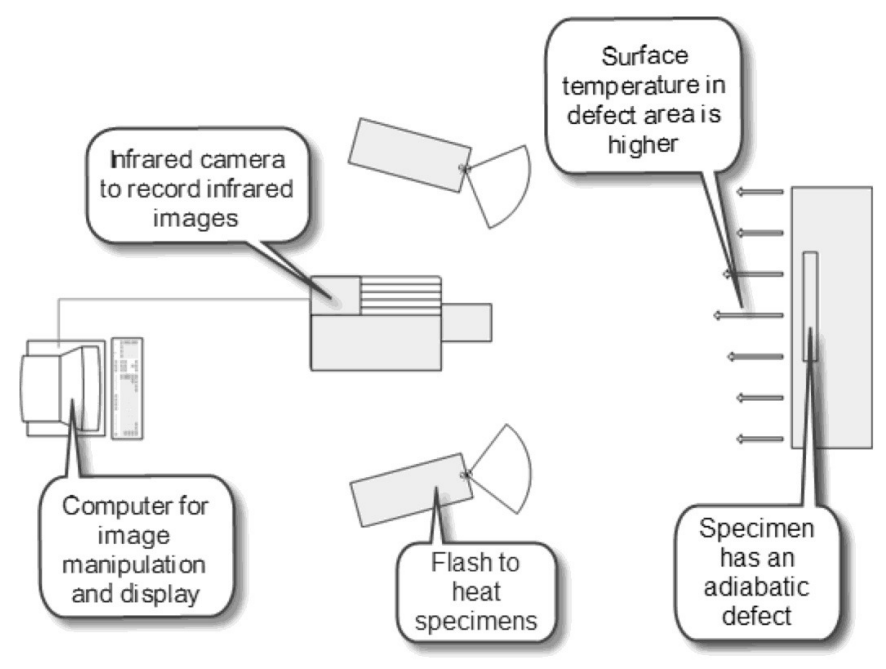

Fig.1 Principle of infrared flash thermography

The initial temperature in the semi-infinite solid $(x \geq 0)$ is $t_{0}$. The solid is heated at its surface $x=0$ by instantaneous plane source of strength $Q$ at time $T=0$, and the surface $x=0$ is thermally insulated when $T>0$, the equations are as follows: [2]

$$
\left\{\begin{array}{l}
\frac{\partial \theta}{\partial \tau}=a \frac{\partial^{2} \theta}{\partial x^{2}} \\
\left.\theta(x, \tau)\right|_{\tau=0}=0, x>0 \\
Q=\rho c \int_{0}^{+\infty} \theta(x, \tau) d x \\
0 \quad=0, \tau>0
\end{array}\right.
$$

When $\tau>0$, the temperature at the surface $x=0$ is

$$
\theta(0, \tau)=\frac{Q}{\rho c(\pi a \tau)^{1 / 2}}
$$

Here $\theta(x, \tau)=t(x, \tau)-t_{0}, \rho$ is mass density, $c$ is heat capacity per mass. $\theta(0, \tau)$ is simplified to $\theta(\tau)$, then we have

$$
\theta(\tau)=\frac{Q}{\rho c(\pi a \tau)^{1 / 2}}
$$

Taking the natural logarithm of both sides of Eq. (6),

$$
\operatorname{In} \theta(\tau)=\operatorname{In}\left[Q /\left(\rho c a^{1 / 2}\right)\right]-0.5 \operatorname{In}(\pi \tau)
$$

In practice, actual logarithmic data may vary from ideal linear behavior for some reasons. However, a polynomial provides an excellent fit to experimental data [3], where

$$
\operatorname{In}(\theta(\tau))=\sum_{i=0}^{N} a_{i}[\operatorname{In}(\tau)]^{i}
$$

First logarithmic derivative can be derived from Eq. (8), and thermogram sequences of first logarithmic derivative can also be obtained. Numerical derivative values could be used to identify defects in the specimen [1]. 


\section{Experiments}

The structure of component from the outside to the inside was aluminum panel-GFRP-foam core (shown in figure 2). Flash thermography testing of debonding defects between panel and GFRP or debonding defects between GFRP and foam core was researched in this paper. A reference standard was designed as shown in figure 3 . The debonding defects were formed in the way that metal sheets were inserted into the specimen before curing and pulled out after curing. The defects in the first line were between panel and GFRP, and the defects in the sixth line were between GFRP and foam core (see figure 3). The flash thermography testing result of reference standard was shown in figure 4.

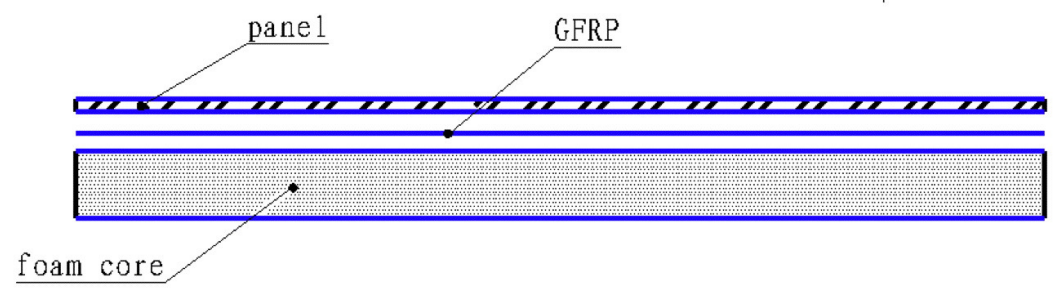

Fig.2 Structure of component

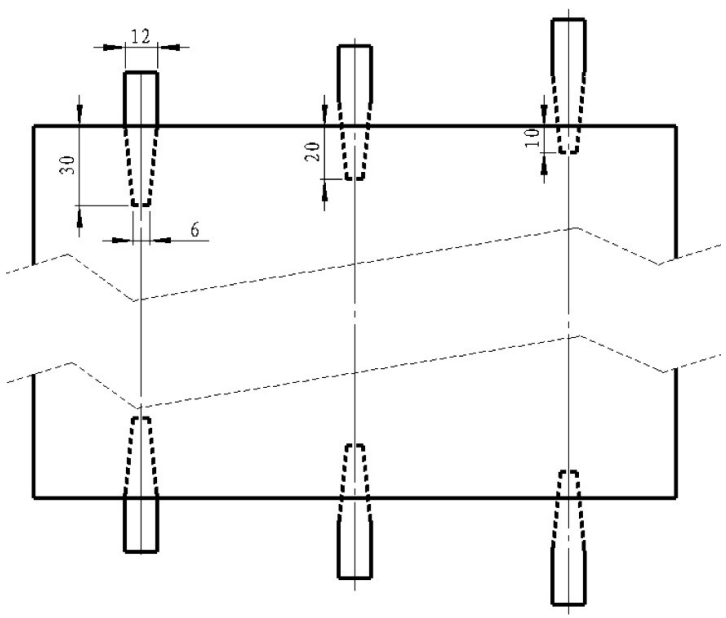

Fig.3 Specimen with artificial debonding defects

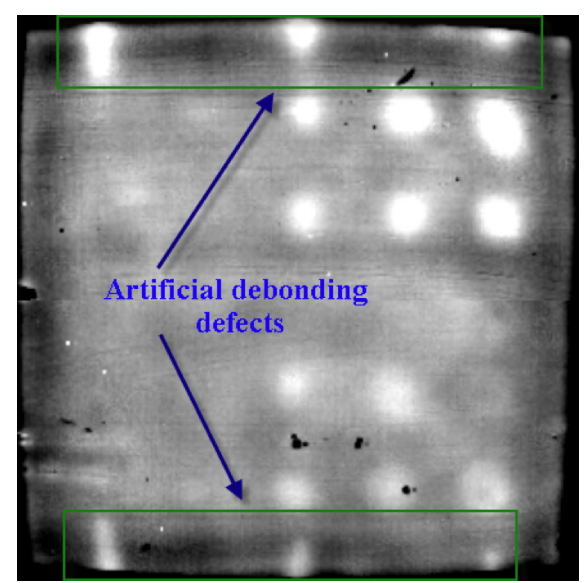

Fig.4 Flash thermography testing result of the reference standard 
The maximum of first logarithmic derivative values of defects in the reference standard at some image was considered as a threshold value which was used to identify defects. The image should be chosen in early time of image sequence recorded by IR camera when influence of lateral heat diffusion is not obvious yet and the edges of defects are clear. If the maximum of first logarithmic derivative values of some suspicious areas was greater than the threshold value, those suspicious areas were defects. The average value of first logarithmic derivative values of flaw-free areas was defined as a basic value. The basic value of the reference standard is often different from the basic value of areas to be detected, and the basic values of different areas to be detected are often different from each other too. So the threshold value used to identify defects should be modified to void misjudgment according to the difference of basic values in the reference standard and that in areas to be detected. An area was tested twice, and it had been painted before testing each time. The basic value of results from first test and second test were obviously different. The image obtained from second test on the right shown in figure 5 was brighter than the one gotten from first test on the left, and these two images were chosen at the same time. If the threshold value used in the first test had been used in the second test, most of the area had been thought of as defects in the second test.

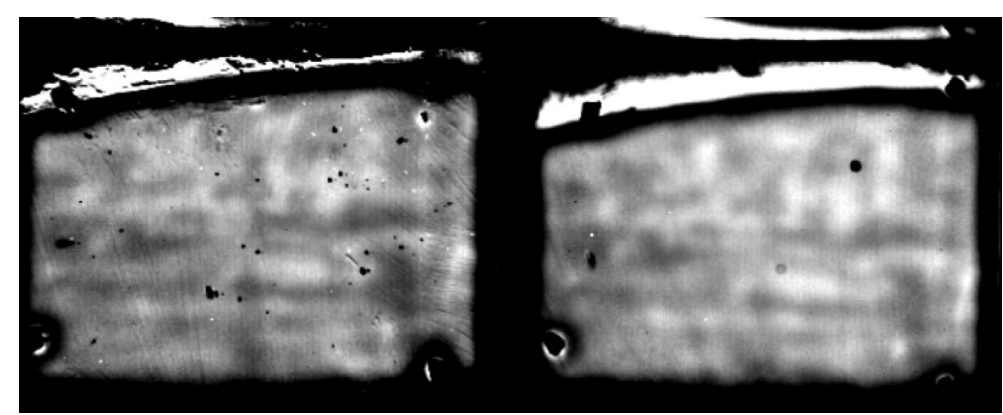

Fig.5 Different basic values of an area obtained from different tests

To prove the accuracy of infrared flash thermograghy, Component 0\# was tested, and results were compared with those from ultrasonic testing. The component was dissected to verify results from infrared flash thermograghy further. 95 percent of debonding defects were found using infrared flash thermograghy from the results of Statistics except severe defects which were not larger than diameter of $10 \mathrm{~mm}$. However, a few small flaws were not found out by ultrasonic testing neither [4]. Furthermore, results from infrared flash thermograghy were more explicit than those from UT, and the edge of defect was legible as shown in figure 6 which was a picture of Area 1-2 and Area 1-3 after being dissected and in figure 7 which was testing result of the same areas.

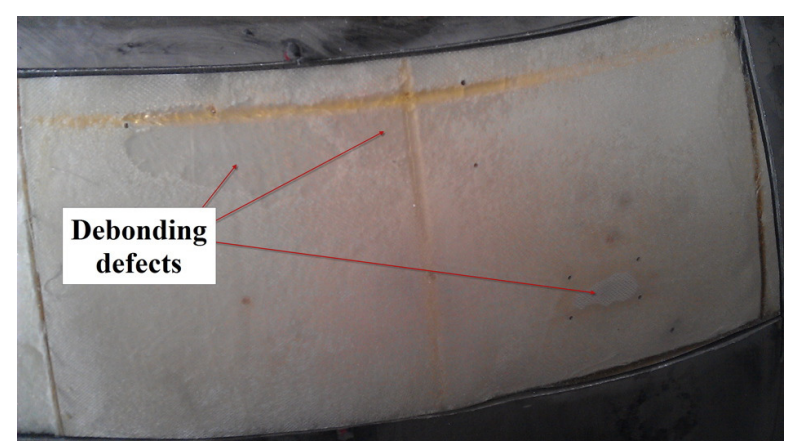

Fig.6 Picture of Area 1-2 and Area 1-3 after being dissected 


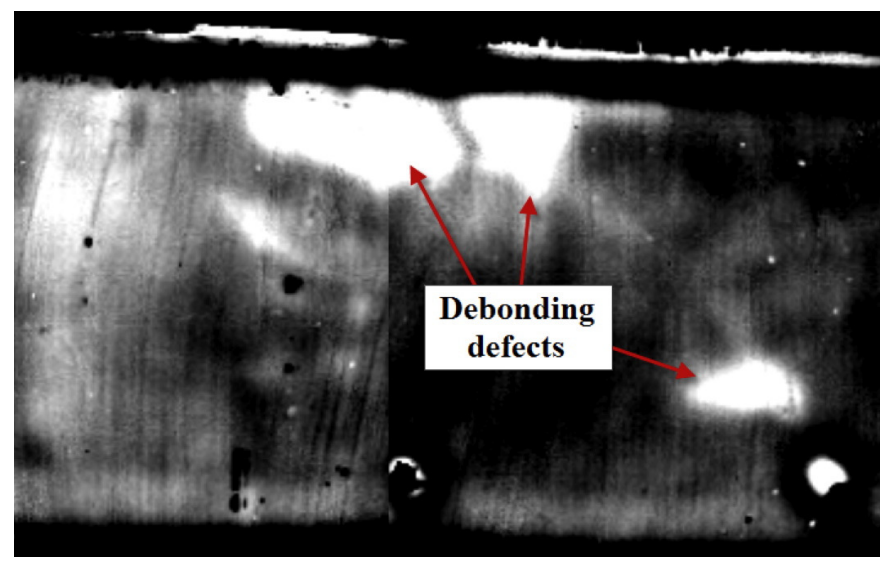

Fig.7 Testing results of Area 1-2 and Area 1-3 using infrared flash thermograghy

Testing results of Component $1 \#$ before and after load test were compared. The debonding defect in Area 1-9 was enlarged, in the middle of which the flaw-free area was narrowed shown in figure 8(the left image was gotten from testing before load test and the right image was obtained from testing after load test).
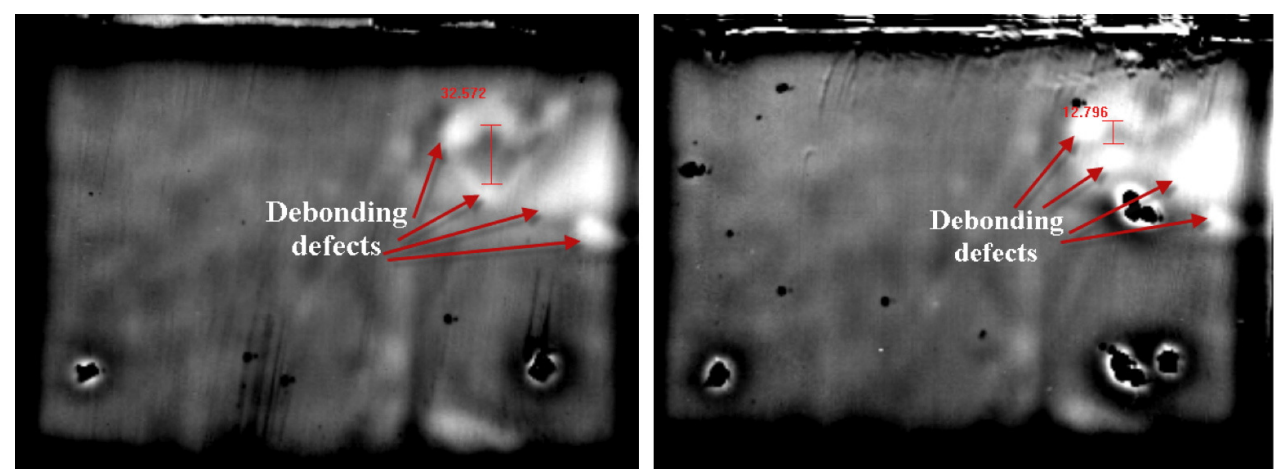

Fig.8 Expansion of the defect in Area 1-9 (left image was gotten from testing before load test and right image was obtained from testing after load test)

\section{Influence factors of defect identification}

There are several factors which affect defect identification such as defect size, defect thickness, defect depth, curve surface of component, black paint thickness and uniformity. Based on our study on modeling and simulation, influence of defect size, defect thickness and defect depth on temperature signals had the following description:

1) Temperature signals increased with size of debonding defect. The larger debonding defect was, the easier it was to be found;

2) Temperature signals increased with thickness of debonding defect.

3) Temperature signals decreased with depth of debonding defect. The shallower debonding defect was, the easier it was to be found;

The first logarithmic derivative value of defects followed the same rules through experiments, so defect size, defect thickness and defect depth should be taken into account in defect identification.

The surface of panel had to be painted black because the panel to be tested was made of aluminum. The different thickness of black paint in local areas on the surface of panel affected heat diffusion from the exterior to the interior and brought about added heat imbalance which disturbed the first logarithmic derivative value locally and marked 
the image as shown in figure 9. Thickness of black paint in the centre area of the image was obviously different from that in the surrounding areas in figure 9, and the first logarithmic derivative value of the centre area was much lower than that of the surrounding areas in result. The significant difference of black paint thickness on the surface disturbed the acquisition of inner structure information and influenced defect identification.

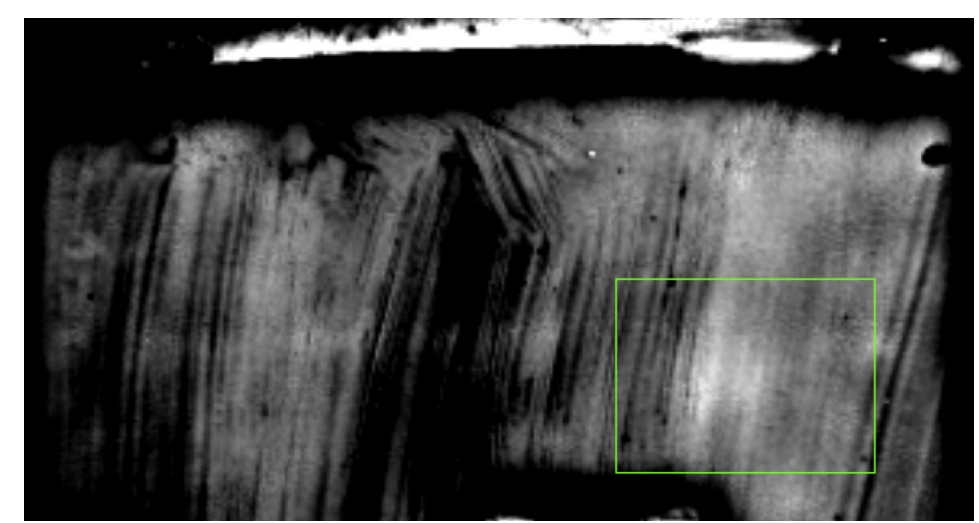

Fig.9 Influence of different thickness of black paint on results

Influence of detection point is more obvious on a curve surface than that on a plane. Difference of detection point and relative position results in: 1) disturbing defect identification because the first logarithmic derivative values of a defect are distinct; 2) distortion of defects in the image and inaccurate measurement of defect size. Two images of a defect obtained from different detection points in different relative positions of detection center were given in figure 10. The first logarithmic derivative value of a defect marked in a red circle in the left image was much lower than that in the right image because the defect was farther to the detection center in the left image than it was in the right image and detection points were different.
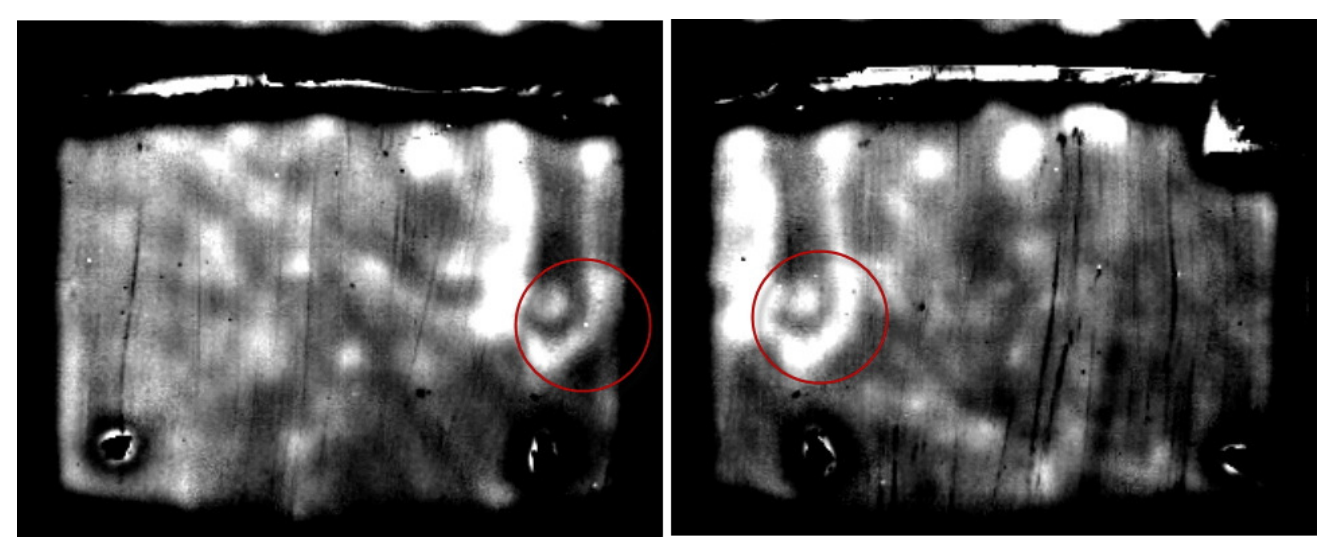

Fig.10 Results of a defect from different detection points

Besides the factors mentioned above, uniformity of testing system and testing environment are also important factors, especially such as uniformity of heat and environmental temperature, which are not discussed in detail in this paper.

\section{Conclusion}

1) The maximum of first logarithmic derivative values of defects in the reference standard at some image was considered as a threshold value which was used to identify defects. The image should be chosen in early time of image 
sequence recorded by IR camera when influence of lateral heat diffusion is not obvious yet and the edges of defects are clear.

2) The average value of first logarithmic derivative values of flaw-free areas was defined as a basic value. The threshold value used to identify defects should be modified to void misjudgment according to the difference of basic values in the reference standard and that in areas to be detected.

3) Results from infrared flash thermograghy were reliable;

4) Results from infrared flash thermograghy were more explicit than those from UT, and edges of defects were legible;

5) There are several factors which affect defect identification such as defect size, defect thickness, defect depth, black paint thickness and uniformity, curve surface of component, uniformity of testing system and testing environment.

\section{References}

[1] ASTM E 2582-07 Standard Practice for Infrared Flash Thermography of Composite Panels and Repair Patches Used in Aerospace Applications, p1341-1346

[2] Conduction of Heat in Solid. Hou Zhen-bing, He Shao-jie, Li Shu-xian. Shanghai Scientific and Technical Publishers, 1984, p22-95

[3] S.M.Shepard. Advances in Pulsed Thermography. Thermosense XXIII, Proceedings of SPIE Vol.4360(2001), p511-515

[4] The Various State-of-art Non-destructive Test Methods for PMI Foam Cored Sandwich Structure. Xu Wei, Meng Yin-Gen, Liu Ji-Chao. The tenth annual meeting of the society for nondestructive testing, China, 2013.09, p210-1214 